\section{Propriedades psicométricas da Satisfaction with Life Scale em adultos jovens brasileiros}

\author{
Psychometric properties of the Satisfaction with \\ Life Scale in young Brazilian adults
}

\section{Propiedades psicométricas de la Satisfaction with Life Scale en adultos jóvenes brasileños}

\author{
Bianca Nubia Souza Silva 1 \\ Lucas Arrais de Campos 2 \\ Wanderson Roberto da Silva 3 \\ João Marôco 4 \\ Juliana Alvares Duarte Bonini Campos 3
}

\section{Resumo}

A Satisfaction with Life Scale (SWLS) foi proposta originalmente na lingua inglesa para medir a percepção geral do indivíduo em relação à vida. $O$ objetivo do estudo é avaliar as propriedades psicométricas da SWLS quando aplicada a indivíduos adultos jovens e estimar a influência de características demográficas na satisfação com a vida. É uma amostra não probabilística de indivíduos adultos jovens (18 a 35 anos) de ambos os sexos de Araraquara, São Paulo, Brasil. Avaliou-se o ajustamento da SWLS aos dados por análise confirmatória, utilizou-se os indices: índice de ajuste comparativo (CFI, em inglês), indice de Tucker-Lewis (TLI, em inglês) e raiz quadrada média residual padronizada (SRMR, em inglês). A confiabilidade foi estimada pelo coeficiente alfa ordinal e ômega. A invariância fatorial foi estimada por análise multigrupos, usando-se a estatística teste da diferença de CFI ( $\triangle C F I)$. A comparação dos escores médios de satisfação com a vida segundo o sexo, a idade, o estrato econômico e o exercício de atividade laboral foi realizada por análise de variância (ANOVA). Participaram 2.170 indivíduos (feminino: 67,8\%; idade: 22,09 anos). O ajustamento do modelo às diferentes amostras foi adequado $(C F I=0,981-0,998 ; T L I=0,962-0,996$; $S R M R=0,026-0,040$; ômega = 0,842-0,869; alfa =0,862-0,889). Observou-se invariância estrita para as variáveis avaliadas. Os dados obtidos com a SWLS foram válidos, confiáveis e invariante entre as amostras de diferentes sexo, idade, estrato econômico e exercício de atividade laboral. A satisfação com a vida foi maior entre os indivíduos de estrato econômico mais elevado, não diferindo segundo sexo, idade e exercício de atividade laboral.

Adulto; Satisfação Pessoal; Psicometria

\author{
Correspondência \\ J. A. D. B. Campos \\ Faculdade de Ciências Farmacêuticas, Universidade Estadual \\ Paulista Júlio de Mesquita Filho. \\ Rod. Araraquara-Jaú, Km 01, s/n, Araraquara, SP \\ 14800-903, Brasil. \\ juliana.campos@unesp.br \\ 1 Faculdade de Odontologia de Araraquara, Universidade \\ Estadual Paulista Júlio de Mesquita Filho, Araraquara, Brasil. \\ 2 Faculty of Medicine and Health Technology, Tampere \\ University, Tampere, Finland. \\ 3 Faculdade de Ciências Farmacêuticas, Universidade Estadual \\ Paulista Júlio de Mesquita Filho, Araraquara, Brasil. \\ 4 William James Centre for Research, Instituto Universitário de \\ Ciências Psicológicas, Sociais e da Vida, Lisboa, Portugal.
}




\section{Introdução}

Nas últimas décadas, a experiência subjetiva tem sido estudada por meio de diversos construtos, entre eles pode-se citar o bem-estar subjetivo ${ }^{1}$. O bem-estar subjetivo se refere à avaliação da vida realizada pelo indivíduo com base em seu próprio conjunto de critérios 2 . Esse construto é formado por um componente afetivo relacionado com a felicidade e um componente cognitivo inerente à satisfação com a vida 3 . O componente afetivo envolve as experiências de emoções, sejam elas prazerosas (afetos positivos) ou não (afetos negativos) 4 . Já o componente cognitivo é o julgamento de como a pessoa avalia a sua própria vida, levando em consideração a vida como um todo ou componentes específicos da mesma 3 . A satisfação com a vida é um produto cognitivo, diante disto, não se pode dizer que ela é uma experiência direta e verificável, nem um fato pessoal conhecido, ela envolve uma comparação entre a situação vivida atualmente pelo indivíduo e os padrões internalizados 3 .

Os principais modelos do bem-estar são apresentados em dois diferentes modelos teóricos denominados bottom-up e top-down. Nas teorias top-down, investiga-se de que forma os fatores internos ou intrínsecos, tais como os valores pessoais, podem influenciar no bem-estar do indivíduo e parte do pressuposto de que as pessoas possuem uma predisposição para interpretar as experiências da própria vida de forma positiva e negativa. Já para os modelos de bottom-up levam-se em consideração como os processos externos, por exemplo, as situações de vida e as variáveis demográficas, podem influenciar no bem-estar 5 .

Nos últimos anos, a preocupação em mensurar o bem-estar subjetivo de indivíduos e populações tem aumentado, contudo, como este trata de um conceito latente (não mensurável diretamente construto) sua medida ainda representa um desafio 1. Para realizar a avaliação desse construto alguns instrumentos são apresentados na literatura, tais como a Subjective Happines Scale (SHS), Escala de Bem-Estar Subjetivo (EBES), a Satisfaction with Life Scale (SWLS), a Oxford Happines Inventory (OHI), a Positive and Negative Affect Schedule-Expanded (PANAS-X) 2.3.4, entre outros. Esses são instrumentos denominados psicométricos, ou seja, construídos com base em métodos padronizados com a finalidade de medir conceitos abstratos, não mensurados diretamente (como, por exemplo, afetos, comportamentos e percepções) 6. Porém, é importante destacar que a escolha do instrumento para a avaliação do bem-estar subjetivo deve estar elencada no contexto de aplicação e na adequação do instrumento escolhido para amostra nos diferentes contextos ${ }^{7}$.

Uma das primeiras escalas confeccionadas para avaliar o bem-estar subjetivo em indivíduos adultos foi a SWLS 8. A SWLS foi desenvolvida por Diener et al. 8 e tem sido muito utilizada, principalmente, por ser uma escala de fácil preenchimento e ter se mostrado válida e confiável em diferentes amostras e contextos 9,10,11. O instrumento foi traduzido em diferentes idiomas: francês 12 , espanhol 13 , holandês 14 , chinês 15 , hebraico 16 , português 17 , alemão 18 e foi empregado em diferentes populações 9,10,19 e em diferentes culturas e nações 2,19 .

A partir da aplicação da SWLS em diferentes contextos, a literatura tem destacado a influência das características demográficas na satisfação com a vida dos indivíduos 10. Alguns estudos apontam relação significativa entre a satisfação com a vida e a idade, o sexo 10, o estrato econômico 20 e o trabalho 21. Em relação à idade, alguns autores observaram que as pessoas mais jovens apresentaram uma posição mais positiva em relação ao bem-estar 22, o mesmo ocorre entre as mulheres 23 . Contudo, essa relação não é consensual, Glaesmer et al. 18 e Vazquez et al. 21 mostraram que nem a idade nem o sexo estavam significativamente associados ao bem-estar em uma amostra representativa de indivíduo adultos da Alemanha e Espanha, respectivamente. Com relação às condições socioeconômicas, Vazquez et al. 21 enfatizaram que as pessoas empregadas relataram uma melhor satisfação com a vida em comparação com as desempregadas.

Visto que a SWLS é um instrumento passível de mensuração de parâmetros psicométricos, é imprescindível avaliar a validade e confiabilidade dos dados recolhidos com o mesmo quando aplicado a uma determinada amostra, pois apenas desta maneira é possível garantir a qualidade da medida que está sendo apresentada 6 . Embora estudos tenham mostrado as propriedades psicométricas da SWLS quando aplicada a indivíduos adultos de diferentes contextos 2,10 , poucas pesquisas avaliaram como o instrumento mede a satisfação com a vida em diferentes subgrupos. Frente ao exposto, este estudo tem como objetivo avaliar as propriedades psicométricas da SWLS quando aplicada a indivíduos adultos jovens e estimar a influência de características demográficas na satisfação com a vida. 


\section{Métodos}

\section{Delineamento de estudo e aspectos éticos}

Trata-se de um estudo observacional do tipo transversal, com amostragem não probabilística delineada por conveniência. $\mathrm{O}$ cálculo do tamanho amostral mínimo foi realizado considerando-se a necessidade de dez respondentes por parâmetro a ser estimado no modelo final 24, para que as abordagens analíticas possam ser adequadamente realizadas. Levando-se em conta que a SWLS apresenta dez parâmetros a serem estimados, o tamanho mínimo de amostra deverá ser de 100 sujeitos por subgrupo de estudo. Ainda, considerando-se uma taxa de perda de 20\%, esse valor deverá ser de 125 sujeitos. Contudo, dado que o objetivo do trabalho foi estudar as propriedades psicométricas da SWLS para a população de adultos jovens, a amostra deve ser suficientemente grande para capturar de forma conveniente a variabilidade populacional. Por esse motivo, optou-se por trabalhar com uma amostra da população de tamanho mais alargado, muito superior às recomendações usuais para a realização dos testes estatísticos 25 .

A amostra foi composta por indivíduos adultos jovens (18 a 35 anos), brasileiros da comunidade de Araraquara, São Paulo, Brasil (amostra normativa), de ambos os sexos. Como critérios de exclusão foram adotados o fato de ser gestante, lactante ou portador de deficiência visual grave que comprometa a leitura dos instrumentos de coleta de dados. Cabe esclarecer que a escolha por trabalhar com indivíduos adultos jovens foi feita pelo fato destes estarem inseridos numa etapa da vida sustentada por anseios e expectativas de construção de suas identidades pessoais e profissionais, assumindo diferentes papéis e responsabilidades, o que torna a avaliação da satisfação com a vida uma oportunidade para entender este processo, pois é nesta fase que os compromissos do papel do adulto gradualmente se cristalizam 26. Para a composição da amostra, inicialmente, os indivíduos foram recrutados na Universidade Estadual Paulista Julio de Mesquita Filho (UNESP) entre os estudantes, funcionários técnico-administrativos e docentes. Em seguida, esses indicaram novos participantes, adotando-se, portanto, o processo de amostragem em bola de neve.

A pesquisa foi divulgada entre os funcionários, estudantes e professores da UNESP (campus de Araraquara) por meio de diferentes meios de comunicação (e-mails, convites pessoais, rede social, entre outros). $\mathrm{O}$ instrumento foi aplicado em formato de papel e foi preenchido pelos participantes, de forma individual, em sala reservada para este fim. Foram convidados a participar 3.063 indivíduos, destes, $8 \%$ não aceitaram o convite. Dos 2.818 que aderiram ao estudo, 23\% desistiram durante o preenchimento dos instrumentos. Assim, a amostra final foi composta por 2.170 indivíduos.

Apenas os participantes que assinaram o Termo de Consentimento Livre e Esclarecido (TCLE) foram incluídos na amostra de estudo. O desenvolvimento deste projeto foi aprovado pelo Comitê de Ética em Pesquisa em Seres Humanos da Faculdade de Odontologia de Araraquara (UNESP) (C.A.A.E 88600318.3.0000.5416). Os dados foram apresentados sequencialmente, de acordo com a declaração Fortalecendo a Comunicação de Estudos Observacionais em Epidemiologia (STROBE) 27.

\section{Caracterização da amostra e variáveis de estudo}

Para a caracterização da amostra foram levantadas informações como sexo, idade, estado civil, trabalho e o estrato econômico dos participantes. Esse estrato foi estimado utilizando-se o Critério Brasil (ABEP) 28. A satisfação com a vida foi estimada usando-se a SWLS descrita a seguir.

\section{Instrumento de medida}

A SWLS foi proposta originalmente na língua inglesa para avaliar a satisfação geral com a própria vida ${ }^{29}$. A escala é unifatorial, composta por cinco itens cujas respostas estão dispostas em escala Likert de 7 pontos (1: discordo totalmente a 7: concordo totalmente). Neste estudo, foi utilizada a versão em português proposta por Gouveia et al. 17. Os cinco itens de avaliação global são: (1) na maioria dos aspectos, minha vida é próxima ao meu ideal; (2) as condições da minha vida são excelentes; (3) estou satisfeito com minha vida; (4) dentro do possível, tenho conseguido as coisas importantes que quero da vida; e (5) se pudesse viver uma segunda vez não mudaria quase nada na minha vida. 


\section{Análise dos indicadores psicométricos}

As medidas resumo (média, mediana e desvio padrão - DP) e de forma da distribuição (assimetria e curtose) foram estimadas com o intuito de verificar a distribuição das respostas dos participantes dadas aos itens do instrumento. Valores absolutos de assimetria e curtose acima de 3 e 7 , respectivamente, foram considerados fortes desvios da normalidade 6,30.

\section{Validade de construto}

Para a avaliação dos indicadores de validade e confiabilidade, a amostra total foi aleatoriamente subdividida em duas partes, sendo denominadas "Amostra Teste" e "Amostra de Validação", e as propriedades psicométricas da SWLS foram avaliadas para as duas amostras separadamente.

Para a verificação do ajustamento do modelo teórico proposto para o instrumento, à amostra de estudo foi realizada a análise fatorial confirmatória (AFC). Foi utilizado o método de estimação média ponderada dos mínimos quadrados e variação ajustada (WLSMV, em inglês). Como índices de avaliação da qualidade do ajustamento do modelo aos dados foram usados o índice de ajuste comparativo (CFI, em inglês), o índice de Tucker-Lewis (TLI, em inglês) e o raiz quadrada média residual padronizada (SRMR, em inglês). O ajustamento foi considerado aceitável quando $\chi^{2} / \mathrm{gl} \leq 5,0$; CFI e TLI $\geq$ 0,90 ; e SRMR $<0,08$ 6. O peso fatorial $(\lambda) \geq 0,50$ foi considerado adequado. Quando o ajustamento do modelo não foi aceitável, foram calculados os índices de modificação usando-se o método dos multiplicadores de Lagrange (ML). Valores de ML > 11 foram inspecionados.

A validade convergente foi avaliada com base na variância extraída média (VEM) 6. A VEM foi estimada utilizando-se a proposta de Fornell \& Larcker 31 e foi considerada adequada se $\geq 0,50$.

As análises foram realizadas com os pacotes lavaan e semTools no programa R (http://www.r-project.org).

\section{Invariância fatorial}

Para avaliar se a proposta fatorial obtida se mantém nas amostras independentes (teste x validação), foi realizada a avaliação da invariância fatorial por meio da análise multigrupos. Foi utilizada a estatística teste da diferença de CFI ( $\Delta \mathrm{CFI})$ para a comparação dos pesos fatoriais $(\lambda)$, limiares $(\mathrm{t})$ e variância/ covariância dos resíduos (Cov/Res). Foram considerados os valores de CFI dos modelos configuracional (M0), do modelo dos pesos fatoriais (M1), do modelo dos thresholds (M2) e do modelo dos resíduos (M3). Atestou-se invariância quando a redução do CFI $(\Delta \mathrm{CFI})$ foi inferior a 0,01 32. O mesmo procedimento foi adotado para a avaliação da invariância entre as subamostras agrupadas segundo as características demográficas idade, sexo, exercício de atividade laboral e nível econômico.

\section{Confiabilidade}

A confiabilidade foi avaliada usando-se as métricas de consistência interna coeficiente alfa ordinal $(\alpha)$ e coeficiente ômega $(\omega)$ calculadas com o pacote semTools do programa R. Os valores $\alpha$ e $\omega \geq 0,7$ foram indicadores de consistência interna satisfatória 6 .

\section{Comparação dos escores de satisfação com a vida}

Os escores de satisfação com a vida foram calculados usando-se a média aritmética das respostas dadas aos itens do modelo da SWLS ajustado aos dados. Para a separação dos subgrupos foram consideradas as características sexo (masculino; feminino), idade ( $<25$ anos; $\geq 25$ anos), exercício de atividade laboral (não; sim) e estrato econômico [baixo: estratos C ( R\$ 2.165,00), D e E (R\$ 768,00); alto: estratos $\mathrm{A}(\mathrm{R} \$ 20.888,00)$ e $\mathrm{B}(\mathrm{R} \$ 7.053,00)]$, estes valores são referentes à renda média domiciliar apresentada para os estratos do Critério Brasil 28. Cabe esclarecer que a definição do ponto de corte para a idade foi realizada após a avaliação da característica dos participantes da amostra de estudo. Como houve predominância de indivíduos em fase universitária, optou-se por trabalhar com o 
percentil 75 das idades na amostra, que representa exatamente a idade de finalização dos estudantes universitários (24 anos). Assim, considerou-se os grupos: $<25$ anos e $\geq 25$ anos.

Após a verificação e a apresentação de evidências que sustentam a existência de invariância forte da SWLS, procedeu-se a comparação entre os escores de satisfação com a vida nessas amostras. Foi utilizada a análise de variância (ANOVA) e o tamanho do efeito foi estimado a partir do $\eta^{2}{ }_{\mathrm{p}}$. Os pressupostos de normalidade e homocedasticidade foram testados e confirmados em todos os subgrupos (assimetria $<3$, curtose $<7$, teste de Levene: $\mathrm{p}>0,05$ ). As comparações múltiplas foram realizadas usando-se o pós-teste de Tukey. O nível de significância adotado foi de $5 \%$.

\section{Resultados}

A amostra foi constituída por 2.170 adultos, sendo a maioria do sexo feminino $(67,9 \%)$. A média de idade foi de 22,09 anos ( $\mathrm{DP}=3,74$ anos) $(78,9 \%<25$ anos e $21,1 \% \geq 25$ anos). Dos participantes, $69,6 \%$ relataram não exercer atividade laboral. Com relação ao nível econômico, $16,6 \%$ pertenciam ao estrato $\mathrm{A}, 46,5 \%$ ao B, $36,5 \%$ ao $\mathrm{C} \mathrm{e} 0,4 \%$ aos estratos $\mathrm{D}$ e $\mathrm{E}$. Cabe esclarecer que nem todos os participantes responderam a todas as informações demográficas (6,4\% não responderam à $\mathrm{ABEP}$ e, portanto, o nível econômico não pôde ser estimado; $2,5 \%$ não responderam se trabalham ou não e apenas uma pessoa não identificou o sexo). Essa ausência de informação não impactou nas análises realizadas.

As medidas resumo das respostas dadas para cada um dos itens são apresentadas na Tabela 1. Nenhum item da SWLS mostrou valor absoluto de assimetria e curtose indicativo de fortes desvios à distribuição normal.

$\mathrm{Na}$ Tabela 2, apresentamos os indicadores psicométricos da SWLS ajustada às diferentes subamostras.

O ajustamento do modelo e a confiabilidade foram adequados para todas as subamostras. Observou-se invariância estrita (forte) entre os modelos para todas as características avaliadas, apontando que a SWLS operacionaliza o conceito satisfação com a vida da mesma maneira nos diferentes subgrupos abrindo, portanto, a possibilidade de comparações entre os mesmos.

Na Tabela 3, mostramos a comparação dos escores médios de satisfação com a vida de acordo com o sexo, a idade, o exercício de atividade laboral e o estrato econômico.

Observou-se diferença significativa na satisfação com a vida entre os diferentes estratos econômi$\cos (\mathrm{p}<0,001)$, sendo que os indivíduos com os maiores estratos apresentaram os maiores escores de satisfação. Essa diferença foi consequência da resposta dada ao item 2 da SWLS (Figura 1).

\section{Tabela 1}

Estatística descritiva das respostas dadas aos itens da Satisfaction with Life Scale (SWLS) pelos participantes.

\begin{tabular}{lccccc}
\hline & $\mathbf{1}$ & $\mathbf{2}$ & Item & $\mathbf{5}$ \\
\hline Média & 4,57 & $\mathbf{3}$ & $\mathbf{4}$ & $\mathbf{5}$ \\
Desvio padrão & 1,59 & 1,59 & 4,97 & 5,19 & 4,26 \\
Assimetria & $-0,55$ & $-0,65$ & $-0,76$ & $-0,86$ & $-0,23$ \\
Curtose & $-0,63$ & $-0,41$ & $-0,12$ & 0,14 & $-1,18$ \\
Mínimo & 1 & 1 & 1 & 1 & 1 \\
Percentil 25 & 3 & 4 & 4 & 4 & 3 \\
Percentil 50 & 5 & 5 & 5 & 6 & 5 \\
Percentil 75 & 6 & 6 & 6 & 6 & 6 \\
Máximo & 7 & 7 & 7 & 7 & 7 \\
\hline
\end{tabular}


Tabela 2

Indicadores psicométricos da Satisfaction with Life Scale (SWLS) para a amostra.

\begin{tabular}{|c|c|c|c|c|c|c|c|c|c|}
\hline \multirow[b]{2}{*}{ Amostra } & \multicolumn{3}{|c|}{ AFC } & \multicolumn{3}{|c|}{ Confiabilidade } & \multicolumn{3}{|c|}{$\Delta \mathrm{CFI}$} \\
\hline & CFI & TLI & SRMR & VEM & $\omega$ & $\mathbf{a}$ & M1-Mo & M2-M1 & M3-M2 \\
\hline Teste & 0,998 & 0,996 & 0,027 & 0,592 & 0,853 & 0,873 & & & \\
\hline Validação & 0,997 & 0,993 & 0,036 & 0,597 & 0,855 & 0,874 & $-0,001$ & 0,002 & 0,000 \\
\hline \multicolumn{10}{|l|}{ Sexo $[n=2.169]$} \\
\hline Masculino & 0,984 & 0,969 & 0,039 & 0,577 & 0,842 & 0,862 & & & \\
\hline Feminino & 0,990 & 0,981 & 0,028 & 0,604 & 0,860 & 0,879 & 0,000 & 0,000 & $-0,002$ \\
\hline \multicolumn{10}{|c|}{ Idade (anos) $[\mathrm{n}=2.170]$} \\
\hline$<25$ & 0,987 & 0,975 & 0,032 & 0,584 & 0,850 & 0,869 & & & \\
\hline$\geq 25$ & 0,994 & 0,987 & 0,026 & 0,630 & 0,869 & 0,889 & 0,000 & 0,001 & $-0,001$ \\
\hline \multicolumn{10}{|l|}{ Trabalho $[n=2.115]$} \\
\hline Não & 0,992 & 0,984 & 0,026 & 0,591 & 0,852 & 0,872 & & & \\
\hline Sim & 0,982 & 0,982 & 0,038 & 0,602 & 0,859 & 0,877 & 0,001 & 0,002 & $-0,002$ \\
\hline \multicolumn{10}{|c|}{ Estrato econômico * $[n=2.030]$} \\
\hline Baixo (C, D e E) & 0,981 & 0,962 & 0,040 & 0,599 & 0,856 & 0,873 & & & \\
\hline Alto $(A$ e $B)$ & 0,990 & 0,979 & 0,030 & 0,586 & 0,851 & 0,870 & 0,001 & 0,002 & $-0,004$ \\
\hline
\end{tabular}

AFC: análise fatorial confirmatória; CFI: índice de ajuste comparativo; M0: modelo configuracional; M1: modelo de limiares; M2: modelo de pesos fatoriais; M3: modelo de resíduos; SRMR: raiz quadrada média residual padronizada; TLI: índice de Tucker-Lewis VEM: variância extraída média; a: coeficiente alfa ordinal; $\triangle \mathrm{CFI}=$ diferença de $\mathrm{CFI}$; $\omega$ : coeficiente ômega.

* Estrato econômico de acordo com o Critério Brasil 28: A (R\$ 20.888,00); B (R\$7.053,00); C ( $R \$ 2.165,00)$, D e E (R\$ 768,00).

Tabela 3

Comparação dos escores médios de satisfação com a vida de acordo com o sexo, a idade, o exercício de atividade laboral (trabalho) e o estrato econômico.

\begin{tabular}{|c|c|c|c|c|c|}
\hline \multirow[t]{2}{*}{ Característica } & \multirow[t]{2}{*}{$\mathbf{n}$} & \multirow[t]{2}{*}{ Média \pm desvio padrão } & \multicolumn{3}{|c|}{ ANOVA } \\
\hline & & & $\mathbf{F}$ & Valor de $p$ & $\eta_{p}^{2}$ \\
\hline \multicolumn{6}{|l|}{ Sexo } \\
\hline Masculino & 697 & $4,79 \pm 1,27$ & & & \\
\hline Feminino & 1.472 & $4,77 \pm 1,30$ & 0,115 & 0,734 & $<0,001$ \\
\hline \multicolumn{6}{|l|}{ Idade (anos) } \\
\hline$<25$ & 1.713 & $4,78 \pm 1,28$ & & & \\
\hline$\geq 25$ & 457 & $4,77 \pm 1,34$ & 0,016 & 0,898 & $<0,001$ \\
\hline \multicolumn{6}{|l|}{ Trabalho } \\
\hline Não & 1.472 & $4,78 \pm 1,29$ & & & \\
\hline Sim & 643 & $4,80 \pm 1,27$ & 0,090 & 0,764 & $<0,001$ \\
\hline \multicolumn{6}{|c|}{ Estrato econômico * } \\
\hline$C, D$ e $E$ & 750 & $4,60 \pm 1,34 a$ & & & \\
\hline B & 943 & $4,80 \pm 1,26 b$ & & & \\
\hline$A$ & 337 & $5,12 \pm 1,13 c$ & 21,759 & $<0,001 * *$ & 0,020 \\
\hline
\end{tabular}

Nota: letras diferentes indicam diferenças estatísticas.

* Estrato econômico de acordo com o Critério Brasil 28: A ( $R \$ 20.888,00)$; $B(R \$ 7.053,00)$;

$C(R \$ 2.165,00)$, D e $E(R \$ 768,00)$.

** Diferença estatística significativa para $p=0,05$. 


\section{Figura 1}

Comparação dos escores médios das respostas dadas aos itens da Satisfaction With Life Scale (SWLS) pelos participantes dos diferentes estratos econômicos.

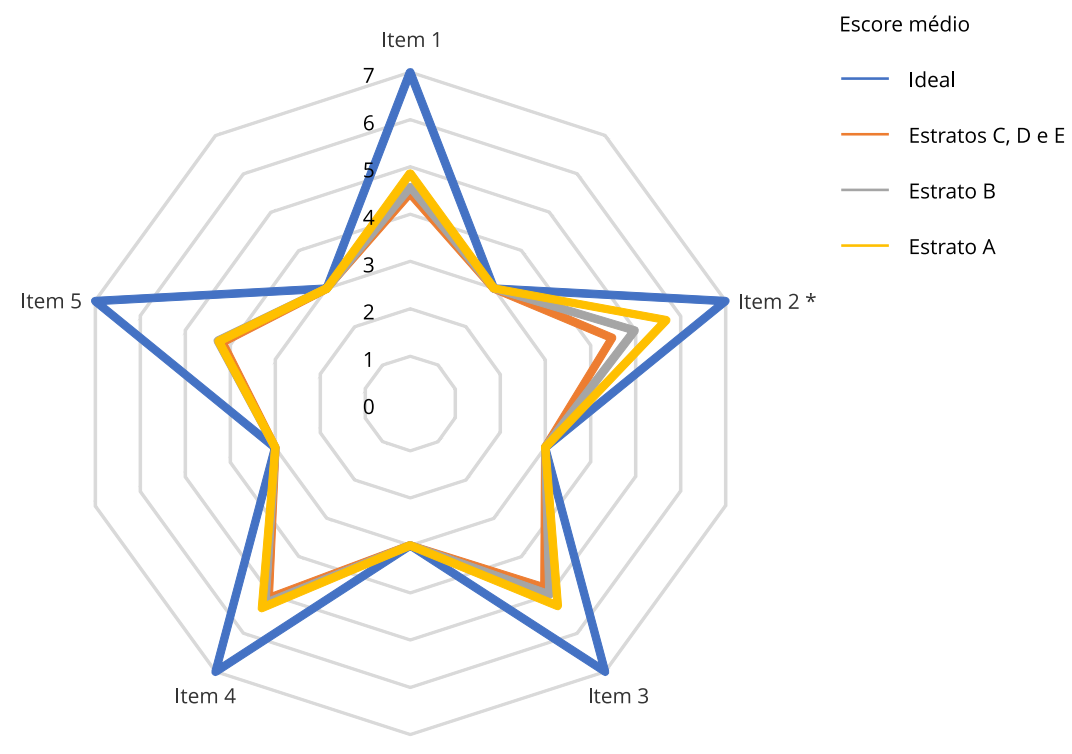

Nota: estrato econômico de acordo com o Critério Brasil 28: A ( $R \$ 20.888,00) ; B(R \$ 7.053,00) ; C(R \$ 2.165,00)$, D e E ( $R \$$

768,00).

* Valor de $p<0,001 ; \eta_{p}^{2}=0,020$.

\section{Discussão}

O presente estudo confirmou a validade e a confiabilidade dos dados para a avaliação do bem-estar subjetivo em adultos. O ajustamento adequado da SWLS aos dados corrobora os resultados apresentados para amostras em diferentes contextos na literatura 29,33.

A SWLS é um dos instrumentos mais utilizados no mundo para medir o bem-estar subjetivo 33 . Embora os estudos científicos tenham apresentado evidências para o uso da SWLS e mostrado boas propriedades psicométricas, incluindo validade e confiabilidade 10,29,33, ainda existem questões importantes que precisam ser abordadas. Uma questão diz respeito à invariância da escala, que embora muitas evidências apoiem o uso em adultos em geral 10,33, poucas pesquisas avaliaram como o instrumento mede a satisfação com a vida em subgrupos 10,34,35.

As conclusões relativas à invariância são inconsistentes na literatura, alguns estudos relataram que a SWLS apresenta operacionalização distinta entre os sexos $34 \mathrm{e}$ as idades 10, e outro relatou que a escala é invariante nestas condições 35 . A falta de invariância apresentada nesses estudos pode ser explicada muitas vezes pelo tamanho inadequado e/ou a composição da amostra 33. Neste estudo, observou-se invariância de medida estrita da SWLS nas diferentes amostras testadas.

A avaliação das diferenças existentes na satisfação com a vida entre indivíduos de diferentes sexos tem sido de grande interesse. Assim, evidências relacionadas à invariância entre os sexos da SWLS torna-se relevante como pressuposto para a utilização do instrumento em ambos os sexos e para viabilizar a realização de comparações diretas entre os sexos. A invariância da SWLS entre os sexos tem sido testada 34,36 , contudo, poucos estudos 19,33,36 avaliaram ou atestaram a invariância estrita da SWLS. As evidências apresentadas pelo presente estudo corroboram os resultados apresentados por Bai et al. 37 e por Tomás et al. ${ }^{19}$, que verificaram invariância estrita da SWLS quando aplicada a homens e mulheres em uma amostra de adultos chineses e angolanos, respectivamente. É importante 
enfatizar a necessidade de condução do processo de validação dos instrumentos utilizados para a mensuração de parâmetros psicométricos e avaliação da invariância entre grupos sempre que apresentar alterações das características amostrais, visto que a validade e a confiabilidade são propriedades dos dados obtidos e não do instrumento por si só 38 e que as comparações entre os grupos só podem ser realizadas se a invariância for confirmada 39.

Além da invariância estrita entre os sexos, observou-se invariância estrita para a idade o que já havia sido relatado apenas por Tomás et al. 19. Com relação à invariância estrita da SWLS entre amostras de diferentes estratos econômicos e exercício de atividade laboral verificada, estas evidências podem ser consideradas um diferencial deste estudo, uma vez que não foram encontrados trabalhos que realizaram estas avaliações. Diante disso, os resultados encontrados contribuem com a ampliação do conhecimento das possibilidades de utilização da SWLS. Considerando que esse é um instrumento curto, de fácil aplicação ou preenchimento em estudos epidemiológicos e clínicos, e que operacionaliza o conceito satisfação com a vida de forma equivalente em subgrupos distintos, abre-se a possibilidade de utilização do mesmo para o rastreamento desta condição em grupos com diferentes características.

Com relação à comparação dos escores médios de satisfação com a vida, não foi verificada diferença entre os indivíduos das duas faixas etárias estudadas. Hinz et al. 10, por sua vez, encontraram diferenças na satisfação com a vida de indivíduos de 50 a 59 anos e os mais jovens, sendo que os primeiros estavam mais insatisfeitos. Desse modo, sugerimos que sejam realizados estudos futuros que possam incluir indivíduos de diferentes fases de desenvolvimento.

Com relação ao exercício de atividade laboral, os resultados encontrados vão ao encontro dos apresentados por Vazquez et al. 21 que verificaram, em amostra espanhola, uma melhor satisfação com a vida entre as pessoas que relataram trabalhar. Pode-se especular que isso esteja relacionado ao fato de incluirmos em nossa amostra apenas adultos jovens com o predomínio de indivíduos que relataram não exercer atividade laboral, o que pode ter minimizado a variabilidade dos dados.

Em relação ao estrato econômico, os resultados sugerem que a renda tem uma relação positiva e significativa com a satisfação com a vida. Esses achados são consistentes com os estudos anteriores 11,20. Apesar disso, a relação entre a renda e a satisfação com a vida deve ser interpretada com cautela, visto que a literatura apresenta uma inconsistência nos achados e aponta que condições culturais e sociais também devem ser consideradas 1 . Um aspecto que tem sido relatado como interferente no bem-estar é a presença da desigualdade social nos países; a literatura mostra que pessoas que vivem em países com muita desigualdade social tendem a se sentir mais insatisfeitas 40,41,42. Diener \& Seligman 43 relataram que a condição financeira é mais importante para os indivíduos que vivem em países pobres, e que o aumento do salário em países ricos como os da Europa e os Estados Unidos não gerou aumento no bem-estar geral dos indivíduos. Para os autores, isso pode ocorrer porque pessoas felizes tendem a concentrar seus ideais em outros objetivos sociais e morais e não somente na condição financeira. Com isso, estudiosos sugerem que o dinheiro é um preditor de bem-estar e satisfação com a vida apenas em países pobres 40,41. Esse fato pode ser observado na Figura 1, em que fica claro que em nossa amostra a satisfação com a vida esteve longe do ideal (valor máximo da escala), destacando a diferença significativa dos escores médios observados para o item 2 ("as condições da minha vida são excelentes") entre os participantes com diferentes níveis econômicos. Pode-se especular que dentro da nossa realidade a interpretação sobre as condições de vida podem ter sido remetidas a condições relacionadas ao conforto e sustento, o que por sua vez depende do estrato econômico do indivíduo. Por esse motivo, esse item apresentou maior capacidade discriminante em relação ao nível econômico do que os demais. Assim, a realização de estudos transnacionais futuros, envolvendo países com diferentes níveis de desenvolvimento, poderá ser interessante no sentido de agregar informações para esta discussão.

Como limitações deste trabalho, pode-se citar o seu desenho de estudo transversal que inviabiliza o estabelecimento de relações de causa e efeito entre as variáveis investigadas, o delineamento amostral não probabilístico que pode limitar a validade externa dos dados para outras amostras e a alta prevalência de estudantes universitários na amostra, o que resultou na apresentação de dados referentes a indivíduos mais jovens ( $75 \%<25$ anos). Destaca-se ainda que essa última limitação também causou impacto na característica do nível econômico da amostra, uma vez que, no Brasil, o acesso à universidade ainda é predominantemente das classes de maior poder econômico. Apesar dessas limitações, 
espera-se que este estudo possa contribuir com profissionais e pesquisadores de diferentes áreas, para o entendimento da satisfação com a vida de indivíduos adultos jovens com diferentes características, permitindo a realização de discussões e reflexões que possam sustentar a elaboração de ações educativas, preventivas e de intervenção voltadas à melhoria da vida das pessoas.

\section{Conclusão}

Os dados obtidos com a SWLS para a amostra de indivíduos adultos foram válidos e confiáveis. Observou-se invariância estrita da SWLS entre as amostras de diferentes sexos, idades, estratos econômicos e exercício de atividade laboral. A satisfação com a vida foi maior entre indivíduos de estrato econômico mais elevado, não diferindo segundo sexo, idade e exercício de atividade laboral.

\section{Colaboradores}

Todos os autores contribuíram significativamente na concepção e desenho do estudo, na coleta, análise e interpretação dos dados, na elaboração do artigo, na revisão crítica do conteúdo intelectual e na aprovação da versão final a ser publicada.

\section{Informações adicionais}

ORCID: Bianca Nubia Souza Silva (0000-00032928-1566); Lucas Arrais de Campos (0000-00031514-5758); Wanderson Roberto da Silva (00000001-8897-8772); João Marôco (0000-0001-92145378); Juliana Alvares Duarte Bonini Campos (0000-0001-7123-5585).

\section{Agradecimentos}

O presente trabalho foi realizado com apoio da Coordenação de Aperfeiçoamento de Pessoal de Nível Superior (CAPES; código de financiamento 001) e da Fundação de Amparo à Pesquisa do Estado de São Paulo (FAPESP; processos \#2017/20315-7, \#2018/06739-1, \#2019/17200-9 e \#2019/24424-0). 


\section{Referências}

1. Machado L. Bem-estar subjetivo: implicações para psiquiatria e para a psicologia médica. Rio de Janeiro: Medbook; 2017.

2. Pavot W, Diener E. The satisfaction with life scale and the emerging construct of life satisfaction. J Posit Psychol 2008; 3:137-52.

3. Diener E. New findings and future directions for subjective well-being research. Am Psychol 2012; 67:590-7.

4. Lyubomirsky S, King LA, Diener. E. The benefits of frequent positive affect: does happiness lead to success? Psychol Bull 2005; 131:80355.

5. Luhmann M, Hofmann W, Eid M, Lucas RE. Subjective well-being and adaptation to life events: a meta-analysis. J Pers Soc Psychol 2012; 102:592-615.

6. Marôco J. Análise de equações estruturais: fundamentos teóricos, software \& aplicações. 2a Ed. Pêro Pinheiro: ReportNumber; 2014.

7. Silva WR, Dias JCR, Pimenta F, Campos JADB. Assessment of the body shape concern: a challenge. Psychology, Community \& Health 2014; 3:103-19.

8. Diener E, Emmons RA, Larsen RJ, Griffin S. The satisfaction with life scale. J Pers Assess 1985; 49:71-5.

9. Emerson SD, Guhn M, Gadermann AM. Measurement invariance of the Satisfaction with Life Scale: reviewing three decades of research. Qual Life Res 2017; 26:2251-64.

10. Hinz A, Conrad I, Schroeter ML, Glaesmer $\mathrm{H}$, Brähler E, Zenger M, et al. Psychometric properties of the Satisfaction with Life Scale (SWLS), derived from a large German community sample. Qual Life Res 2018; 27:1661-70.

11. Diener E. The remarkable changes in the science of subjective well-being. Perspect Psychol Sci 2013; 8:663-6.

12. Blais MR, Vallerand RJ, Pelletier LG, Brière NM. L'échelle de satisfaction de vie: validation canadienne-française du 'Satisfaction with Life Scale'. Can J Behav Sci 1989; 21:210.

13. Pons D, Atienza FL, Balaguer I, García-Merita ML. Satisfaction with life scale: analysis of factorial invariance for adolescents and elderly persons. Percept Mot Skills 2000; 91:62-8.

14. Arrindell WA, Heesink J, Feij JA. The Satisfaction with Life Scale (SWLS): appraisal with 1700 healthy young adults in the Netherlands. Pers Individ Dif 1999; 26:815-26.

15. Sachs J. Validation of the satisfaction with life scale in a sample of Hong Kong university students. Psychologia 2003; 46:225-34.

16. Anaby D, Jarus T, Zumbo BD. Psychometric evaluation of the Hebrew language version of the Satisfaction with Life Scale. Soc Indic Res 2010; 96:267-74.
17. Gouveia VV, Milfont TL, Fonseca PN, Coelho JAPM. Life satisfaction in Brazil: testing the psychometric properties of the Satisfaction with Life Scale (SWLS) in five Brazilian samples. Soc Indic Res 2009; 90:267-77.

18. Glaesmer H, Grande G, Braehler E, Roth M. The German version of the Satisfaction with Life Scale (SWLS). Eur J Psychol Assess 2011; 27:127-32.

19. Tomás J, Gutiérrez M, Sancho P, Romero I. Measurement invariance of the Satisfaction with Life Scale (SWLS) by gender and age in Angola. Pers Indiv Dif 2015; 85:182-6.

20. Yun YH, Rhee YE, Kang E, Sim JA. The Satisfaction with Life Scale and the Subjective Well-Being Inventory in the general Korean population: psychometric properties and normative data. Int J Environ Res Public Health 2019; 16:1538.

21. Vazquez C, Duque A, Hervas G. Satisfaction with Life Scale in a representative sample of Spanish adults: validation and normative data. Span J Psychol 2013; 16:E82.

22. Lehmann BA, Bos A, Rijken M, Cardol M, Peters GJ, Kok G, et al. Ageing with an intellectual disability: the impact of personal resources on well-being. J Intellect Disabil Res 2013; 57:1068-78.

23. Graham C, Chattopadhyay S. Gender and well-being around the world. International Journal of Happiness and Development 2013; 1:212-32.

24. Hair JF, Black WC, Babin BJ, Anderson RE, Tatham RL. Multivariate data analysis: a global perspective. Upper Saddle River: Prentice Hall; 2009.

25. Campos JADB, Marôco J. Maslach Burnout inventory-student survey: Portugal-Brazil crosscultural adaptation. Rev Saúde Pública 2012; 46:816-24.

26. Papaila DE, Feldman RD. Desenvolvimento físico e cognitivo no início da vida adulta e no adulto jovem. In: Papaila DE, Feldman RD, organizadores. Desenvolvimento humano. 12a Ed. Porto Alegre: AMGH Editora; 2013. p. 450-81.

27. von Elm E, Altman DG, Egger M, Pocock SJ, Gøtzsche PC, Vandenbroucke JP, et al. The Strengthening the Reporting of Observational Studies in Epidemiology (STROBE) statement: guidelines for reporting observational studies. J Clin Epidemiol 2008; 61:344-9.

28. Associação Brasileira de Empresas de Pesquisa. Critério de Classificação Econômica Brasil 2018. São Paulo: Associação Brasileira de Empresas de Pesquisa; 2018. 
29. Diener E, Emmons RA, Larsen RJ, Griffin S. The satisfaction with Life Scale. J Pers Assess 1985; 49:71-5.

30. Finney SJ, DiStefano C. Non-normal and categorical data in structural equation modeling. In: Hancock GR, Mueller RO, editors. Structural equation modeling: a second course. 2nd Ed. Charlotte: Information Age Publishing; 2006. p. 439-92.

31. Fornell C, Larcker DF. Evaluating structural equation models with unobservable variables and measurement error. Journal of Marketing Research 1981; 18:39-50.

32. Cheung GW, Rensvold RB. Evaluating goodness-of-fit indexes for testing measurement invariance. Struct Equ Modeling 2002; 9:23355.

33. Clench-Aas J, Nes RB, Dalgard OS, Aarø LE. Dimensionality and measurement invariance in the Satisfaction with Life Scale in Norway. Qual Life Res 2011; 20:1307-17.

34. Atienza FL, Balaguer I, García-Merita ML. Satisfaction with life scale: analysis of factorial invariance across sexes. Pers Individ Dif 2003; 35:1255-60.

35. Zweig JS. Are women happier than men? Evidence from the Gallup World Poll. J Happiness Stud 2015; 16:515-41.
36. Shevlin M, Brunsden V, Miles J. Satisfaction with life scale: analysis of factorial invariance, mean structures and reliability. Pers Individ Dif 1998; 25:911-6.

37. Bai X, Wu C, Zheng R, Ren X. The psychometric evaluation of the Satisfaction with Life Scale using a nationally representative sample of China. J Happiness Stud 2011; 12:183-97.

38. Silva WR, Dias JCR, Marôco J, Campos JADB. Confirmatory factor analysis of different versions of the Body Shape Questionnaire applied to Brazilian university students. Body Image 2014; 11:384-90.

39. Millsap RE, Yun-Tein J. Assessing factorial invariance in ordered-categorical measures. Multivariate Behav Res 2004; 39:479-515.

40. Diener E, Biswas-Diener R. Will money increase subjective well-being? Soc Indic Res 2002; 57:119-69.

41. Suh EM, Oishi S. Subjective well-being across cultures. Online Readings in Psychology and Culture 2002; 10. https://scholarworks.gvsu. edu/orpc/vol10/iss 1/1/.

42. Oshio T, Kobayashi M. Income inequality, perceived happiness, and self-rated health: evidence from nationwide surveys in Japan. Soc Sci Med 2010; 70:1358-66.

43. Diener E, Seligman ME. Beyond money: toward an economy of well-being. Psychol Sci Public Interest 2004; 5:1-31. 


\section{Abstract}

The Satisfaction with Life Scale (SWLS) was originally proposed in the English language to measure the individual's overall perception in relation to life. The study aims to evaluate the psychometric properties of SWLS when applied to young adults and estimate the influence of demographic characteristics on life satisfaction, in a non-probabilistic sample of young adult individuals (18 to 35 years) of both sexes in Araraquara, São Paulo State, Brazil. We assessed the fit of SWLS to the data by confirmatory analysis, using the comparative fit index (CFI), Tucker-Lewis index (TLI), and standardized root mean square residual (SRMR). Reliability was estimated by the alpha ordinal coefficient and omega. Factor invariance was estimated by multigroup analysis, with CFI test of statistical difference ( $\triangle C F I)$. Comparison of the mean scores on satisfaction with life according to sex, age, economic stratum, and employment status was performed with analysis of variance (ANOVA). Participation included 2,170 individuals (females: 67.8\%; age: 22.09 years). The model's fit to the different samples was adequate $(C F I=0.981-0.998 ; T L I=0.962-0.996$; $S R M R=0.026-0.040 ;$ omega $=0.842-0.869 ; \mathrm{al}-$ pha $=0.862-0.889$ ). Strict invariance was seen for the target variables. Life satisfaction was greater among individuals in higher economic strata. The data obtained with SWLS were valid, reliable, and invariant between samples with different sex, age, economic strata, and employment status. Life satisfaction was greater among individuals from higher economic strata and did not differ by sex, age, or employment status.

Adult; Personal Satisfaction; Psychometrics

\section{Resumen}

La Satisfaction with Life Scale (SWLS) se propuso originalmente en lengua inglesa para medir la percepción general del individuo en relación con la vida. El objetivo del estudio es evaluar las propiedades psicométricas de la SWLS, cuando se aplica a individuos adultos jóvenes, así como estimar la influencia de las características demográficas en la satisfacción con la vida. Se trata de una muestra no-probabilística de individuos adultos jóvenes (18 a 35 años) de ambos sexos de Araraquara, São Paulo, Brasil. Se evaluó el ajuste del SWLS a los datos por análisis confirmatorio, se utilizaron los indices: indice de ajuste compartimentado (CFI, en inglés), indice de Tucker-Lewis (TLI, en inglés) y raiz cuadrada media estandarizada residual (SRMR, en inglés). La confiabilidad se estimó por el coeficiente alfa ordinal y omega. La invariancia factorial se estimó por análisis multigrupos, utilizando la estadística test de la diferencia de CFI ( $\triangle C F I)$. La comparación de las puntuaciones medias de satisfacción con la vida según: sexo, edad, estrato económico y ejercicio de actividad laboral, se realizó mediante el análisis de variancia (ANOVA). Participaron 2.170 individuos (sexo femenino: 67,8\%; edad: 22,09 años). El ajuste del modelo a las diferentes muestras fue adecuado $(C F I=$ $0,981-0,998 ; T L I=0,962-0,996 ; S R M R=0,026-$ 0,040 ; omega $=0,842-0,869$; alfa $=0,862-0,889$ ). Se observó invariancia estricta para las variables evaluadas. La satisfacción con la vida fue mayor entre los individuos de mayor estrato económico. Los datos obtenidos con la SWLS fueron válidos, confiables e invariantes entre muestras de diferentes sexo, edad, estrato económico y ejercicio de actividad laboral. La satisfacción con la vida fue mayor entre individuos de estrato económico más elevado, no difiriendo según sexo, edad y ejercicio de actividad laboral.

Adulto; Satisfacción Personal; Psicometría
Recebido em 15/Jun/2020

Versão final reapresentada em 29/Ago/2020

Aprovado em 04/Set/2020 